\title{
A Utilização De Objetivos De Desempenho De Operações Como Vetor Para Decisões De Investimentos
}

\section{The Use Of Operations Performance Objectives As A Vector For The Investment Decisions}

\author{
Nilton Cezar Carraro ${ }^{1}$, Carlos Roberto Camello Lima ${ }^{2}$ \\ ${ }^{1}$ Universidade Federal de São Carlos, UFSCAR, Brasil, ${ }^{2}$ Universidade Metodista de Piracicaba, UNIMEP, Brasil. \\ Correspondência: Nilton Cezar Carraro. Rod. Lauri Simões de Barros km 12, Aracaçu CEP 18.290-000, Buri, SP, \\ Brasil. Telefone: +55 (15) 3356-9000. E-mail: nilton.carraro@ufms.br.
}

Recebido: 08 de junho de 2017 Aceito: 18 de outubro de 2018 Publicado: 28 de dezembro de 2018

DOI: http://dx.doi.org/10.21714/1679-18272018v16n2.p145-153

\begin{abstract}
Resumo
A análise convencional de investimentos em ativo fixo ocorre por meio de indicadores predominantemente financeiros. A proposta deste artigo é criar uma forma alternativa para complementar esta análise a partir da utilização dos objetivos de desempenho de operações aliado a métodos qualitativos e quantitativos. Para tanto foi feita uma revisão teórica para observar o que a literatura considera como satisfatório e as lacunas apontadas neste contexto. Com base nesta revisão foi possível criar uma proposta de análise que permite alterar os resultados econômicos e financeiros a partir da correlação de variáveis denominadas como quesitos que poderão influenciar o desempenho tanto positiva como negativamente. Os produtos desta proposta é a elaboração de uma matriz e posteriormente a sua inserção num fluxograma de análise que permite ampliar a visão sobre índices de viabilidade financeira em investimentos para ativos fixos. Como contribuições futuras espera-se a produção de pesquisas no campo prático bem como a produção de softwares que repliquem a análise e a sequencia estabelecida ou até mesmo melhorada.
\end{abstract}

Palavras-chave: Análise Financeira, Análise Econômica, Tomada de decisão.

\begin{abstract}
Conventional analysis of investments in fixed assets occurs predominantly through financial indicators. The purpose of this article is to create an alternative way to complement this analysis from the use of performance objectives operations combined with qualitative and quantitative methods. Therefore a theoretical review was made to watch what the literature considers to be satisfactory and the gaps identified in this context. Based on this review it was possible to create a proposal of analysis that allows you to change the economic and financial results from the correlation of variables termed as questions that may influence the performance both positively and negatively. The products of this proposal is to develop a matrix and then their inclusion in an analysis flow chart that allows broader view of financial viability rates in investments to fixed assets. As future contributions expected to produce research in the practical field and the production of software that replicate analysis and established or even improved sequence.
\end{abstract}

Keywords: Financial Analysis, Economic analysis, Decision making.

Esta obra está licenciada sob uma Licença Creative Commons Attribution 3.0.

\section{Introdução}

A necessidade de novos investimentos faz parte do dia a dia das empresas. Projetos demandam dispêndios em maior ou menor proporção conforme a aquisição. Investimentos em ativos fixos fazem parte desse contexto e a grande preocupação dos investidores é o retorno em termos financeiros e principalmente em relação ao tempo de espera.

Parte dessa preocupação origina-se nos métodos utilizados para análise de projetos de investimentos denominados de índices de viabilidade financeira, priorizados pelo valor presente líquido (VPL), taxa interna de retorno (TIR) e tempo de retorno (PB) (OSBORNE, 2010). 
Estes índices são eficazes quando se tem premissas confiáveis para projeção de receitas e gastos do projeto, tais como um mercado consolidado, cenário econômico estável, produtos baseados em tecnologia sustentada, entre outros. Entretanto, em cenários adversos estes índices mostram-se pouco eficientes, pois a fragilidade do cenário gera instabilidade no projeto e consequentemente nos resultados que não serão suportados conforme apresentado pela pesquisa de Osdagli (2012).

A própria evolução das técnicas de construção de projetos permitiram que outros índices viessem fazer parte dessa análise. Isso se deu principalmente com o surgimento guia do corpo de conhecimentos da administração de projetos (PMBOK) elaborado pelo Project Management Institute (PMI), Nele, além das análises tradicionais defendidas por Damodaran (2001), Copeland, Koller e Jack (2002), Assaf Neto (2007), surgiram também índices de análise de sensibilidade e de cenários, índices de eficiência, eficácia e produtividade.

Aliado ao desejo de constante evolução, este trabalho tem o objetivo de contribuir significativamente a maneira de pensar e agir na avaliação de um projeto de investimentos em ativo fixo, fazendo com que haja opções de escolha e que as decisões sejam tomadas não apenas com base na rentabilidade financeira, mas que para tanto sejam analisadas alternativas oriundas da estratégia de operações.

Para tanto, define-se como problema de pesquisa a seguinte questão: Os objetivos de desempenho de operações são capazes de nortear alternativas de investimentos em ativos fixos? A justificativa para esta questão está na condição de que muitas empresas precisam ter um olhar voltado a alternativas de investimentos em ativos fixos, principalmente no tocante a decisão que não deverá ser tomada exclusivamente com base nos índices tradicionais de viabilidade financeira sob pena de excluir do desempenho elementos como flexibilidade, confiabilidade, velocidade e qualidade.

Buscando alcançar o objetivo proposto e responder a questão levantada, este trabalhado está estruturado em cinco seções, sendo a primeira esta breve contextualização do atual cenário, a segunda seção composta de uma revisão da literatura como forma de fundamentar os métodos de pesquisa que serão apresentados na terceira seção e que demonstrarão as análises, resultados e considerações demonstrados nas últimas seções.

\section{Fundamentação Teórica}

Com o objetivo de embasar a discussão sobre a proposta deste trabalho, esta seção fornece um arcabouço de conhecimento para a fundamentação e posicionamento, onde segundo Ross (1993), a distinção clássica dos objetivos dos projetos de investimento em ativos fixos é: de substituição (reposição do equipamento em uso), modernização (racionalização do modo de produção), e de expansão de capacidade (aumento da oferta de produtos).

Devido ao caráter de irreversibilidade das imobilizações, o custo de oportunidade atrelado à decisão de investimento é um custo real e permite sugerir este investimento como uma sinalização onerosa da existência de oportunidades de crescimento da empresa (BORGONOVO; PECCATI, 2006).

Outra razão para novos investimentos em imobilizações se dá devido à grande necessidade de novos produtos, $\mathrm{o}$ que faz com o que ciclo de vida seja cada vez menor. Segundo Beach et al. (2000), no passado, a necessidade por novos produtos era menor e, consequentemente, o ciclo de vida era maior, requerendo menos investimentos em equipamentos substitutos.

Dessa forma, procurou-se identificar, ao nível das teorias e métodos existentes, quais são os meios para se gerar um fluxo de informações relacionadas à tomada de decisão de investimentos em ativos fixos e seus resultados financeiros e operacionais, principalmente com o objetivo de gerar benefícios competitivos.

Essa pesquisa bibliográfica limitou-se a teorias financeiras, que envolvem os índices de viabilidade financeira como TIR, VPL, PB e FDC, e não financeiras, que envolvem objetivos de desempenho de operações (ODO).

\section{1 Índices De Viabilidade Financeira}

Buscando aumentar o valor das empresas (maximização do lucro) o administrador financeiro em sua área deverá primar sempre por decisões que visem melhorar a rentabilidade da empresa. São várias as situações que levam o administrador a tomar decisões. As mais complexas e importantes são aquelas que envolvem a aplicação e a captação de recursos em decisões de longo prazo. Sendo assim, antes de investir é necessário buscar informações sobre o retorno ao longo do tempo. Neste cenário os indicadores mais utilizados são VPL, TIR e PB.

Valor presente líquido (VPL) é uma tradução da sigla inglesa Net Present Value (NPV), que, segundo Samanez (2002), é um método que tem como finalidade valorar monetariamente em termos de valor presente o impacto dos eventos futuros associados a um projeto ou alternativa de investimento, ou seja, mede o valor presente dos fluxos 
de caixa gerados pelo projeto ao longo da sua vida útil.

Esse exemplo pode ser traduzido na equação do VPL, que segundo Assaf Neto (2007, p. 357), é representada conforme a Equação 1 onde $F C_{t}$ representa os fluxos (benefício) de caixa de cada período, $\mathrm{K}$ representa taxa de desconto do projeto, representada pela rentabilidade mínima requerida, $I_{0}$ representa o investimento processado no momento zero e $I_{t}$ representa o valor do investimento previsto em cada período subsequente.

Equação 1: Valor presente líquido

$$
N P V=\left[\sum_{t=1}^{n} \frac{F C_{t}}{(1+K)^{t}}\right]-\left[I_{0}+\sum_{t=1}^{n} \frac{I_{t}}{(1+K)^{t}}\right]
$$

Segundo Brigham e Ehrardt (2012), existem três condições para identificar que um VPL positivo seja fruto de melhorias oriundas de investimentos em ativos fixos, sendo elas a identificação do porque um projeto possui um VPL projetado positivo, que investimentos com VPL positivo não acontecem por acaso e principalmente qual o tempo de vida útil das melhorias promovidas pelos investimentos. Se o projeto não demonstrar essas três condições, talvez o VPL real não seja positivo.

Magni (2002) confirmou a utilização e aceitação do VPL pelo mercado e meio acadêmico e demonstrou o seu valor em conjunto com o valor econômico agregado (EVA - Economic Value Added). Osborne (2010) promoveu uma nova equação a partir de uma discussão centenária sobre a utilização do VPL. Batista et al. (2011) pesquisaram sobre a utilização do VPL na determinação do valor incremental do mercado de carbono nos projetos de geração de energia elétrica no Brasil e chegaram à conclusão de sua eficácia. Chiu e Escalante (2012) demonstraram um novo modelo para financiamentos com capital externo utilizando o VPL. Frezatti et al. (2013) pesquisaram as decisões de investimento em ativos de longo prazo nas empresas brasileiras e também constataram a utilização do VPL.

Observando estes resultados percebe-se que em determinados momentos o VPL é considerado como satisfatório no seu estado original, em outros a uma busca por um aprimoramento e obtenção de novas respostas. É importante observar que o VPL trabalha com expressão numérica financeira, em investimentos com valores diferentes, os resultados trazidos a valor presente também serão diferentes. Assim, buscando corrigir essa distorção outro índice comumente e associadamente utilização é aquele que auxilia na decisão é a apuração da taxa de retorno do investimento, também conhecida como TIR, que é uma tradução da sigla inglesa Internal Rate of Return (IRR).

Segundo Assaf Neto (2007, p. 348), a TIR representada pela Equação 2, parte do pressuposto que todos os movimentos de caixa são trazidos para o momento zero, onde $I_{0}$ representa o montante do investimento no momento zero (início do projeto), $I_{t}$ representa os montantes previstos de investimentos em cada momento subsequente, $\mathrm{K}$ representa taxa de rentabilidade equivalente periódica (IRR) e $F C$ os fluxos previstos de entradas de caixa em cada período de vida do projeto (benefícios de caixa).

Equação 2: Taxa interna de retorno

$$
I_{0}+\sum_{t=1}^{n} \frac{I_{t}}{(1+K)^{t}}=\sum_{t=1}^{n} \frac{F C_{t}}{(1+K)^{t}}
$$

Segundo Samanez (2002) a TIR pode ser utilizada como taxa interna de retorno de um projeto, taxa máxima de custo de capital de um projeto e taxa de desconto para os fluxos de caixa de um projeto. Ao utilizar a TIR, partese do princípio que se ela for maior do que o custo de capital deve-se aceitar o projeto analogamente.

Segundo Ross, Westerfield e Jaffe (1993) existem várias cautelas ao se aplicar esse método de decisão, porém as mais importantes são para fluxos de caixa não convencionais a taxa será indeterminada em função da mudança de sinais, somente será considerada verdadeira quando os fluxos intermediários puderem ser reinvestidos pela própria TIR e que o projeto deverá ser aceito se a TIR for maior que a taxa desejada.

Bucko (2010) testou a combinação de vários métodos de análise de investimentos e, entre eles, estavam presentes a TIR e a TIRM, demonstrando seu valor de análise para o processo de valuation (criação de valor) e tomada de decisão. Nader, Tomi e Passos (2012) detectaram a utilidade da TIR na análise de investimentos de uma mineradora, avaliando-os até o término da vida útil da jazida. Abensur (2012) identificou as deficiências da TIR em relação a projetos mutuamente excludentes, testando um modelo matemático multiobjetivo em contra posição a sua utilização, demonstrando a ineficácia da utilização da TIR quando utilizada isoladamente.

E para finalizar a sequencia dos índices de viabilidade financeira mais utilizados em projetos de investimentos em ativos fixos apresenta-se o tempo de retorno, que segundo Samanez (2002) é o intervalo de tempo exato para que a empresa recupere seu investimento inicial a partir das entradas de caixa em um determinado projeto. 
Por ser de fácil compreensão, este método vem sendo utilizado a muito tempo conforme pesquisas realizadas por Fensterselfer, Galesne e Ziegelmann (1987), confirmaram que grandes empresas brasileiras utilizavam esse método desde a década de 70. Fensterselfer e Saul (1993), replicaram a pesquisa uma década depois e chegaram às mesmas considerações. Gregório (2010) também cita a preocupação com o elemento tempo e demonstra a necessidade de aferição em sua tese para projetos de investimentos em imóveis corporativos.

Entretanto, Kim (2006) relembra em seu trabalho que em países onde a inflação é significativa o PB não pode ser aplicado em seu formato original, devendo ser aplicado o conceito financeiro do desconto, pois a inflação aliada ao tempo de projeto poderá corroer o retorno e assim modificar o tempo de retorno.

Constatadas estas condições na revisão bibliográfica o que permite inferir que estes métodos tradicionais por si só não abrangem questões defendidas por Slack \& Lewis (2002) como sendo responsáveis por uma condição que gera benefícios competitivos e até mesmo vantagens competitivas sustentáveis em um estágio mais avançado, a próxima seção destina-se a apresentar tais elementos.

\subsection{Objetivos De Desempenho De Operações}

Com o propósito de fundamentar aspectos econômicos e operacionais que corroborem com os objetivos desse trabalho, a partir desse tópico, serão apresentados elementos que proporcionarão uma reflexão sobre como a estratégia de operações poderá influenciar na decisão de novos investimentos para o AI.

Slack e Lewis (2002) defendem que para se chegar a um desempenho capaz de surpreender o mercado, a contribuição do AI deve ir além do retorno financeiro. Como forma de mensurar as contribuições advindas desse investimento para o AI, foram eleitos para este trabalho os cinco objetivos de desempenho de operações que partem dos seguintes efeitos demonstrados no Quadro 1.

Ressaltasse que custos é o único dos objetivos de desempenho contemplado pelos índices de viabilidade financeira tratados na seção anterior. Portanto, os demais objetivos de desempenho devem ser tratados de uma forma que não corresponda inicialmente aos ganhos financeiros, mas sim aos ganhos operacionais que poderá proporcionar as alternativas de investimento em ativos fixos.

Quadro 1: Objetivos de desempenho de Operações.

\begin{tabular}{|c|c|c|}
\hline \multicolumn{3}{|c|}{$\begin{array}{r}\text { EFEITOS INTERNOS E EXTERNOS I } \\
\text { DESEMPENHO }\end{array}$} \\
\hline $\begin{array}{l}\text { Beneficios Internos } \\
\text { Potenciais }\end{array}$ & $\begin{array}{l}\text { Objetivos de } \\
\text { Desempenho }\end{array}$ & $\begin{array}{l}\text { Beneficios Externos } \\
\text { Potenciais }\end{array}$ \\
\hline $\begin{array}{l}\text { - Processos sem erros } \\
\text { - Menos complexidade } \\
\text { - Custos mais baixo }\end{array}$ & Qualidade & $\begin{array}{l}\text { - Produtos e serviços sem erros } \\
\text { - Produtos e serviços confiâveis } \\
\text { - Especificação de alto nível }\end{array}$ \\
\hline $\begin{array}{l}\text { - Menos filas elou estoques } \\
\text { - Tempo de processamento mais } \\
\text { rápidos }\end{array}$ & Velocidade & $\begin{array}{l}\text { - Curtos tempos de entrega } \\
\text { - Resposta râpida às solicitações }\end{array}$ \\
\hline $\begin{array}{l}\text { - Maior confiabilidade na operação } \\
\text { - Menos contingências necessárias } \\
\text { - Mais estabilidade interna }\end{array}$ & Confiabilidade & $\begin{array}{l}\text { - Entrega/chegada de produtos e } \\
\text { serviços pontual } \\
\text { - Conhecimento dos tempos de } \\
\text { entrega }\end{array}$ \\
\hline $\begin{array}{l}\text { - Melhor resposta a eventos } \\
\text { imprevistos } \\
\text { - Melhor resposta a variedade de } \\
\text { atividades }\end{array}$ & Flexibilidade & $\begin{array}{l}\text { - Novos produtos e serviços com } \\
\text { freqūência } \\
\text { - Ampla variedade de produtos e } \\
\text { serviços } \\
\text { - Ajustes de volume e entrega }\end{array}$ \\
\hline $\begin{array}{l}\text {-Processos produtivos } \\
\text { - Margens mais altas }\end{array}$ & Custos & - Baixos preços \\
\hline
\end{tabular}

Fonte: Slack e Lewis (2002).

Garvin (1993) já afirmava que as estratégias de produção deveriam estar focadas em diferenciais competitivos, como custos (inicias, operação e manutenção), qualidade (durabilidade, percepção do cliente), flexibilidade (capacidade de mudança de volume, produtos e processos), entrega e serviços.

Resumindo os atributos que o objetivo de desempenho qualidade deve possuir, na aquisição de um ativo fixo, Gaither e Frazier (2001) definem que o mesmo deve ter a capacidade de informar a qualidade aos clientes por meio das melhorias em relação aos produtos pela aparência, redução de índices de mau funcionamento ou defeito e capacidade de duração.

Davis, Aquilano e Chase (2001) defendem que a velocidade adquirida com o novo AI permitirá dois macros 
objetivos de desempenho, que são: a formação de estoques e a entrega no tempo certo. Como formação de estoques, muito embora existam técnicas para minimização desse item, pois entende-se que consomem recursos por conta da ociosidade (JIT), ainda assim, mesmo em menor número, serão necessários para um número elevado de atividades empresariais. Nesse sentido, esses autores afirmam que a velocidade permitirá maiores índices de estoque, maiores índices de produção, métodos de entrega rápida, promessas mais realísticas, melhor controle de produção de encomendas e melhores sistemas de informação.

Carazas (2011) afirma que a confiabilidade está associada intrinsecamente à confiabilidade do equipamento, que tende a ser menor ao longo de sua vida operacional, mesmo com toda a excelência em manutenção que possa ser aplicada, ficado latente que a condição do AI interfere plenamente na confiabilidade das operações.

Gaither e Frazier (2001) afirmam que a flexibilidade de produção tem que ser capaz de responder rapidamente as necessidades dos clientes. Para isso, é fundamental que equipamentos e máquinas permitam duas condições: a flexibilidade do produto e a flexibilidade do volume. Para os autores, a flexibilidade do produto consiste em identificar a capacidade que o sistema de produção tem de mudar rapidamente a produção de um produto para outro. Já a flexibilidade de volume indica a capacidade de aumentar ou reduzir o volume de produção sem que para tanto aumente os custos fixos na mesma proporção. Na aquisição do ativo fixo a flexibilidade é um objetivo de desempenho de operações cuja avaliação dependerá do fluxo de operações atuais e futuros.

Finalizando a revisão sobre os objetivos de desempenho de operações, Porter (1986) afirma que competir por custos é gerar vantagens competitivas em relação aos concorrentes com níveis inferiores de custos. Nesse sentido, Barney e Hesterly (2011) afirmam que indústrias maiores conseguem essa vantagem adquirindo ativos fixos que empresas menores não conseguiriam adquirir.

Esse conjunto de objetivos de desempenho que integra a importância da estratégia de operações está comprovado nas pesquisas de Correa, Paiva e Primo (2010), que levantaram as principais publicações nacionais ocorridas entre 1990 e 2009. Dessa forma, essa pesquisa justifica a importância da observação desses elementos nas aquisições de AI.

Nieweglowski et al. (2010), Rosenzweig, Laseterb e Rothc (2011) e Jayaswal, Jewkes e Ray (2011), defenderam que uma das alternativas é valer-se da engenharia econômica e da estratégia de operações, principalmente no tocante a novos investimentos, para a redução de custos de pré-produção, produção e atividades pós-produção, buscando atender às expectativas dos clientes ao nível de produtos e preços que devam ser fornecidos.

Parece assim claro que a relação das prioridades competitivas passa pelos objetivos de desempenho de operações na aquisição de ativos fixos. Assim, o próximo passo deste trabalho é demonstrar as etapas de decisão, demonstrando a viabilidade e aplicabilidade dos objetivos de desempenho na análise de novos ativos fixos.

\section{Metodologia Aplicada}

Em complemento a utilização de índices de viabilidade financeira citados na seção 2.1, esta pesquisa propôs-se a analisar a concepção de uma maneira diferente de avaliar investimentos em ativo fixo.

Para tanto, utilizou-se da pesquisa descritiva, apoiando em variáveis genéricas com o delineamento correlacional que segundo Appolinário (2011) busca verificar a relação entre as variáveis pesquisadas, que no caso serão os objetivos de desempenho de operações e a interferência nos resultados obtidos por VPL, TIR e PB. Neste método, embora a validade interna seja baixa, ainda não é nula, pois poderá explicar a relação de casualidade. O que permitirá um melhor resultado é a análise e a quantidade de variáveis utilizada pelo sistema.

Para validação das variáveis e dos objetivos de desempenho de operações é necessário a aplicação de testes estatísticos conforme Tabela 1, iniciando pelo Alfa de Cronbach que é um coeficiente que varia entre 0 e 1 e que tem por objetivo evidenciar a proximidade de quesitos que mensuram o mesmo fator. Com esse objetivo, será utilizado o indicador de consistência interna (ou de confiabilidade) Alfa de Cronbach e analisadas as correlações entre os itens e entre cada item e o total do quesito.

Lattin, Carroll e Green (2011, p. 103), propõe em complemento ao Alfa de Cronbach a análise fatorial exploratória que tem por objetivo identificar os fatores comuns (separados dos fatores específicos) e explicar sua relação com os dados observados. Via de regra, utiliza-se uma matriz das cargas fatoriais para demonstrar as correlações entre as variáveis originais e os fatores comuns. a utilização das cargas fatoriais permitem calcular a proporção da variação em cada teste explicada pelos fatores comuns, o que é chamado de comunalidade.

Para este tipo de tratamento, Appolinário (2011) sugere a análise de regressão que é um conjunto de técnicas estatísticas que tem por objetivo determinar a capacidade que uma variável possui de prever o comportamento de outra. Por exemplo: pode-se determinar a renda de um conjunto de sujeitos (variável dependente) a partir do conhecimento do seu nível educacional (variável independente). Difere da correlação na medida em que esta última 
não avalia a causalidade entre variáveis.

E para finalizar utiliza-se a análise por score utiliza as medidas de posição para gerar um ranking dos resultados obtidos na amostra.

Tabela 1: Matriz de análise qualitativa e quantitativa.

\begin{tabular}{|l|l|l|l|l|l|}
\hline \multicolumn{1}{|c|}{ Itens } & Qualidade & Velocidade & Confiabilidade & Flexibilidade & Custo \\
\hline $\mathrm{n} 1$ & & & & & \\
\hline $\mathrm{n} 2$ & & & & & \\
\hline $\mathrm{n} \infty$ & & & & \\
\hline Variância explicada por cada fator & & & \\
\hline Total da variância explicada & & \\
\hline Alfa de Cronbach de cada fator \\
Alfa de Cronbach de escala global \\
\hline Análise de regressão/correlação \\
\hline Análise por score
\end{tabular}

Fonte: elaborada pelos autores

Denominou-se como matriz de interferência, pois a mesma deverá causar ou não reflexos sobre a sequencia de análise de investimentos em ativos fixos. Para tanto na coluna itens deverão ser inseridos os elementos que devam ser parâmetros como desempenho para cada um dos cinco objetivos listados nas colunas laterais. Logo, a quantidade de itens deverá ser igual para cada objetivo para que a análise de regressão/correlação possa ser realizada.

Definidos os testes para análise de interferência dos objetivos de operações nos resultados do projeto, é o momento de analisar a viabilidade financeira e comparar os resultados.

Vale lembrar que os testes estatísticos ora apresentados, bem como outros métodos estocásticos servirão para análise de probabilidade de interferência dos objetivos de desempenho de operações nos resultados econômicos e financeiros, que modificarão as análises obtidas por VPL, TIR e PB, além é claro da decisão. Para tanto, a próxima seção destina-se propõe uma sequencia entre a análise dos dados e a decisão.

\section{Análise Dos Resultados}

Nesta fase, com os dados recolhidos, será realizada uma análise fatorial exploratória e será verificada a confiabilidade e unidimensionalidade de cada um dos cinco quesitos. Resumidamente, para a análise fatorial exploratória, será utilizado o método dos componentes principais com rotação oblimin, de forma a permitir a existência de correlação entre os fatores. Deverá ser analisado cada resultado individualmente e também o total de variância explicada (HAIR et al., 2010).

Para avaliar a confiabilidade e unidimensionalidade dos fatores obtidos, será utilizado o indicador de consistência interna (ou de confiabilidade) Alfa de Cronbach e analisadas as correlações entre os itens e entre cada item e o total do quesito. É fundamental que seja calculo o Alfa de Cronbach sem o item/quesito.

Para finalizar a análise deverão ser calculados scores para cada quesito através da mediana das pontuações de cada um. Será também calculado um score total com a mediana das pontuações de todos os itens/quesitos. Deverão ser analisadas as correlações entre os scores dos cinco quesitos, utilizando-se para o efeito o Coeficiente de Correlação de Pearson e regressão.

Poderá haver a manipulação de cada variável de forma que um item ou quesito não seja estático durante a avaliação, reportando-se a condições de mudanças ao longo da vida útil do investimento. Assim, tem-se com maior propriedade os reflexos que um ativo fixo poderá sofrer ao longo do tempo por questões já abordadas na seção 2 .

Após as análises preliminares através dos objetivos de desempenho de operações ter-se-á os quesitos que mais influenciarão o desempenho por meio dos objetivos almejados, fazendo com que uma análise incorpore a outra, permitindo assim a projeção mais fiel de resultados econômicos e financeiros, pois problemas como atrasos nas entregas (confiabilidade), devoluções por defeitos (qualidade), perda de mercado por incapacidade de mudança de 
configuração de fábrica (flexibilidade) entre outros, certamente aumentarão os resultados negativos.

Feitas estas considerações, é necessário estabelecer uma sequencia para esta análise poderá ser obtida por meio da Figura 2. Observa-se que pelo fluxograma apresentado tem seu inicio na definição dos objetivos de desempenho de operações, passando pela avaliação proposta na matriz apresentada na Tabela 1, para somente então migrar para a análise tradicional por meio dos índices de viabilidade financeira mencionados na seção 2.1.

Figura 2: Sequencia para análise de investimentos.

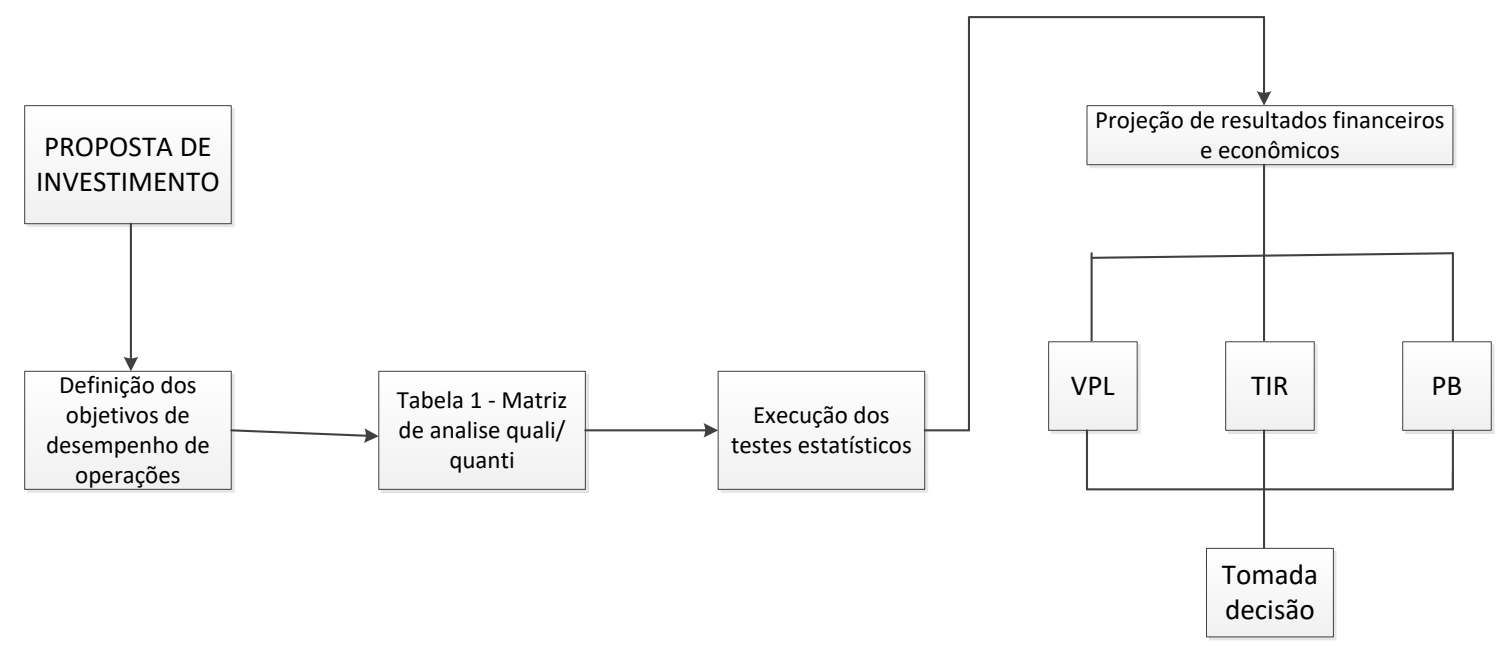

Fonte: elaborada pelos autores

Ressalta-se que a sequencia proposta pela Figura 2 refere-se ao momento exclusivo de analise de viabilidade financeira do projeto, como forma de orientar a correta analise da concepção dos fluxos de caixa a serem obtidos, considerando os fatores positivos e negativos que a inobservância e a falta de reflexão dos objetivos de desempenho de operações podem ser gerados a partir da aquisição de um novo ativo fixo.

\section{Considerações Finais}

Com o objetivo de contribuir significativamente na maneira de pensar e agir na avaliação de um projeto de investimentos em ativo fixo, foi apresentado uma proposta de utilização de métodos qualitativos e quantitativos apropriados a análise de um investimento com o objetivo de interpretar os objetivos de desempenho de operações, propiciando um novo fluxo para análise bem como resultados econômicos e financeiros que originariamente não poderiam ter sido vinculados a análise tradicional envolvendo os índices VPL, TIR e PB.

Constatou-se ao longo da seção 2 deste trabalho, que o problema não está nos índices, mas sim na forma como são concebidos os projetos e como são projetados os resultados. Dessa forma, a inserção dos objetivos de desempenho de operações nas análises fará com que os interessados reflitam sobre as preferências e consequências dos objetivos de desempenho nas operações e consequentemente nos resultados econômicos e financeiros.

Pode-se afirmar que a sequencia sugerida na Tabela 1 são contribuições originais oriundas de métodos quantitativos já consagrados pelo mercado e que até então apareciam na literatura encapsulados a outros requisitos do projeto, mas nunca como contraponto a uma decisão de viabilidade financeira. Sendo assim, a Figura 2 gera um novo status quo na forma de pensar e agir antes de uma decisão de investimento em ativo fixo.

Como contribuições futuras, espera-se que outros pesquisadores ampliem esta forma de pensar e apresentem sugestões de métodos quantitativos, sequencia de análise e até mesmo a criação de softwares de análises já com esta concepção.

\section{Referências}

ABENSUR, E. O. Um modelo multiobjetivo de otimização aplicado ao processo de orçamento de capital. Gestão e Produção, São Carlos, v.19, n.4, out./dez. 2012.

APPOLINÁRIO, F. Dicionário de metodologia científica. 2. ed. São Paulo: Atlas, 2011.

ASSAF NETO, A. Finanças corporativas e valor. 3. ed. São Paulo: Atlas, 2007. 
BATISTA, F.R.S.; et al. Avaliação dos métodos de Grant, Vora \& Weeks e dos mínimos quadrados na determinação do valor incremental do mercado de carbono nos projetos de geração de energia elétrica no Brasil. Pesquisa Operacional. v.31 n.1 Rio de Janeiro Jan./Apr. 2011

BARNEY, J. B.; HESTERLY, W. S. Administração estratégica e vantagem competitiva. 3. ed. São Paulo: Pearson Prentice Hall, 2011.

BEACH, R.; MUHLEMANN, A. P.; PRICE, D. H. R.; PATERSON, A.; SHARP, J. A. A review of manufacturing flexibility. European Journal of Operational Research, v. 122, n. 1, p. 41-57, 2000.

BORGONOVO, E.; PECCATI, L. The importance of assumptions in investment evaluation. International Journal of Production Economics, v. 101, p. 298, 2006.

BRIGHAM, E. F.; EHRHARDT, M. C. Financial management: Theory \& practice. Cengage Learning, 2013.

BUCKO, J. integration model of the financial analysis methods of investiments projects. The engineering economist. v. 55, p. 60-70, 2010.

CARAZAS, F.J.G. Decisões baseadas em risco: método aplicado na indústria de geração de energia elétrica para a seleção de equipamentos críticos e políticas de manutenção. 2011. 218 fls. Tese (Doutorado em Engenharia). Escola Politécnica - Usp, São Paulo, 2011.

CHIU, S.S; ESCALANTE, E.F.G. A companion for NPV: the generalized relative rate of return. The Engineering Economist. v.57, n.3, 2012.

COPELAND, T.; KOLLER, T.; JACK, M. Avaliação de empresas: valuation, calculando e gerenciando o valor das empresas. 3. ed. São Paulo: Makron Books, 2002.

CORREA, H. L.; PAIVA, E. L.; PRIMO, M. A. M. A pesquisa em gestão de operações no Brasil: um breve relato de sua evolução. Revista RAE (eletrônica), v. 9, n. 2. jul/dez. 2010.

DAMODARAN, A. Corporate finance. 2. ed. New York: John Wiley, 2001.

DAVIS, M. M.; AQUILANO, N. J.; CHASE, R. B. Fundamentos da administração da produção. Tradução: Eduardo D'Agord Schaan. 3. ed. Porto Alegre: Bookman Editora, 2001.

FENSTERSELFER, J. E.; GALESNE, A.; ZIEGELMANN, J. A utilização de técnicas analíticas nas decisões de investimento de capital nas grandes empresas no Brasil. Revista de Administração. São Paulo. n. 22 (4) p.70-78, out-dez, 1987.

FENSTERSELFER, J. E.; SAUL, N. Investimento de capital nas grandes empresas. Revista de Administração. São Paulo. n. 28 (3), p. 3-12, jul-set, 1993.

FREZATTI, F. et al. Decisões de investimento em ativos de longo prazo nas empresas brasileiras: qual a aderência ao modelo teórico?. Revista de Administração Contemporânea, Curitiba, v. 16, n. 1, p. 1-22, jan./fev. 2012. Disponível em: <http://www.scielo.br/pdf/rac/v16n1/a02v16n1.pdf〉. Acesso em: 14 set. 2013.

GAITHER, N.; FRAZIER, G. Administração da produção e operações. 8. ed. São Paulo: Cengage Learning, 2001.

GARVIN, D. A. Manufacturing strategic planning. Management Review, California, v. 12, n.2., 1993.

GREGÓRIO, C.A.G. Método para análise de oportunidade de imobilização em imóveis corporativos. Tese. Usp. 2010

HAIR, J. F.; BLACK, W. C.; BABIN, B. J.; ANDERSON, R. E. Multivariate data analisy. 7. ed. Englewood Cliffs: Prentice Hall, 2010.

JAYASWAL, S.; JEWKES, E.; RAY, S. Product differentiation and operations strategy in a capacitated environment. European Journal of Operational Research, v. 210, n. 3, p.716-728, 2011.

KIM, D. Capital budgeting for new projects: on the role of auditing in information acquisition. Journal of Accounting Economics, v. 41, p.257, 2006.

LATTIN, J. M.; CARROLL, J. D.; GREEN, P. E. Análise de dados multivariados. São Paulo: Cengage Learning, 2011.

MAGNI, C. A. Investment decisions in the theory of finance: Some antinomies and inconsistencies. European 
Journal of Operational Research v. 137 p. 206-217, 2002.

NADER, B; TOMI, G. de; PASSOS, A. O. Indicadores-chave de desempenho e a gestão integrada da mineração. REM. Ver. Escola Minas. v. 65(4) p. 537-542, 2012

NIEWEGLOWSKI, R. et al. Desenvolvendo um processo de análise de investimentos baseado em competências. Gestão e Produção, São Carlos, v. 17, n. 2, p. 317-337, 2010.

PORTER, M. E. Estratégia competitiva: técnicas para análise de indústrias e da concorrência. 7. ed. Tradução de Elizabeth Maria de Pinho Braga; Revisão técnica Jorge A. Garcia Gomez. Rio de Janeiro: Campus, 1986.

OSBORNE, M. J. A resolution to the NPV - IRR debate?. The Quarterly Review of Economics and Finance, v. 50, n. 2, p. 234-239, May, 2010.

OZDAGLI, A. K. Financial leverage, corporate investments, and stocks returns. The Review of Financial Studies, v. 25, n. 4, p. 1033-1069, Jan, 2012.

ROSENZWEIG, E. D.; LASETERB, T. M.; ROTHC, A. V. Through the service operations strategy looking glass: influence of industrial sector, ownership and service offerings on B2B e-marketplace failures. Journal of Operations Management, v.29, n. 1, p. 33-48, 2011.

ROSS, S. A.; WESTERFIELD, R. W.; JAFFE, J. F. Corporate Finance, Richard D. Irwin, Boston: 1993.

SAMANEZ, C. P. Matemática financeira: aplicações à análise de investimentos. São Paulo: Prentice Hall, 2002.

LEWIS, M.; SLACK, N. Operations strategy. Prentice-Hall, 2002. 